\title{
Neue Entwicklungen und Einsatzbereiche elektrodialytischer Membranverfahren
}

Dipl.-Ing. F. Kuppinger (Vortragender), Dipl.-Ing. H.-J.-Rapp und Prof. Dr.-Ing. G. Eigenberger, Institut für Chemische Verfahrenstechnik der Universität Stuttgart, Böblinger Str. 72, 70199 Stuttgart.

Durch die zunehmende kommerzielle Verfügbarkeit von polymeren Ionenaustauschermembranen für unterschiedliche Einsatzbereiche erlangen elektrodialytische Membranverfahren zunehmend an Interesse und Bedeutung. Sie eignen sich zur Entfernung von Ionen aus Frisch- und Abwasser, zur Umsalzung von salzhaltigen Lösungen, zur Salzspaltung in wäßrigen und nichtwäßrigen Medien bzw. zur Rückführung von Salzen oder den aus ihnen gewinnbaren Säuren und Laugen in geschlossenen Produktionskreisläufen.

Neben den praxisbewährten Verfahren der Elektrodialyse zur Entsalzung von Lösungen und der Diffusionsdialyse zur Abtrennung starker Säuren aus Salzlösungen haben in den letzten Jahren insbesondere zwei Verfahrensvarianten der Elektrodialyse für eine große Erweiterung des Einsatzbereiches elektrodialytischer Membranverfahren gesorgt:

- die Elektrodialyse mit bipolaren Membranen, die eine - im Vergleich zur Elektrolyse - deutlich energiegünstigere Salzspaltung in die entsprechende Säure und Lauge gestattet;

- die Elektrodialyse mit ionenleitenden Spacern (Harzen) bzw. die kontinuierliche elektrodialytische Regenerierung von Ionenaustauscherharzen, die die Beschränkung des Einsatzbereiches der Elektrodialyse durch die sog. Grenzstromdichte weitgehend aufhebt und die nahezu vollständige elektrodialytische Ionenentfernung bis hin zu sehr niedrigen Konzentrationen möglich macht. Im Vergleich zur konventionellen Regenerierung wird hierbei der Bedarf an Regenerierchemikalien eingespart.

Im Rahmen des Beitrages wird die Elektrodialyse mit bipolaren Membranen und die elektrodialytische Regenerierung von Ionenaustauscherharzen vorgestellt und diskutiert. Anhand konkreter Anwendungsbeispiele wird auf die Vorteile aber auch auf die Grenzen dieser Verfahren eingegangen. Die Beispiele betreffen im Falle der Elektrodialyse mi bipolaren Membranen die geschlossene Kreislaufführung im Bereich der Säuren- und Laugenwäsche sowie den Einsatz bipolarer Membranen zur chemischen Synthese in nichtwäßrigen Medien. Die elektrodialytische Regenerierung von Ionenaustauscherharzen wird an den Beispielen der Schwermetallrückgewinnung aus Galvanikspülwässern und der Abtrennung von Anionen aus wäßriger Lösung diskutiert.

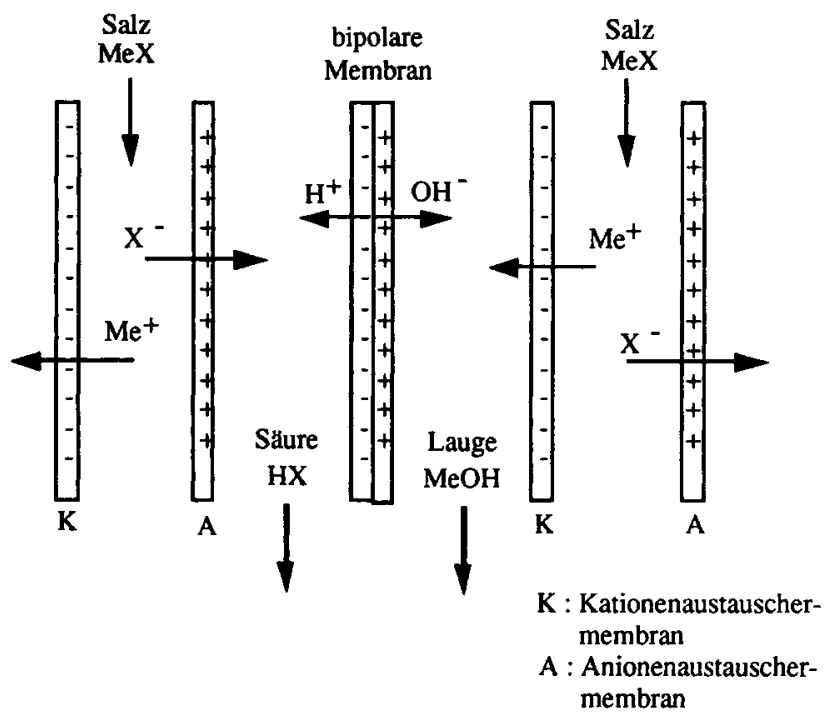

Abb. 1. Prinzip der Umwandlung eines Salzes in die entsprechende Säure und Base mit Hilfe der Elektrodialyse mit bipolaren Membranen. 